\title{
Produtividade de linhagens avançadas de amendoim em condições de sequeiro no Nordeste brasileiro
}

\author{
Roseane C. Santos ${ }^{1 *}$, Gizelda M. Rêgo ${ }^{2}$, Astrogildo P. G. da Silva ${ }^{3}$, José O. L. Vasconcelos ${ }^{3}$, \\ João L. B. Coutinho 4 Péricles de A. Melo Filho 5
}

\begin{abstract}
RESUMO
O nze linhagens avançadas de amendoim, eretas e rasteiras, foram conduzidas em condições de sequeiro, em quatro estados nordestinos, visando-se avaliar a produtividade em vagens e sementes. 0 s ensaios foram conduzidos no período de 2005 a 2007, nos municípios de Araripina e Parnamirim, PE, Lagarto, SE, Cruz das Almas e Caetité, BA, Campina G rande e Itabaiana, PB. 0 delineamento experimental adotado foi em blocos ao acaso, com 13 tratamentos, constituídos de oito linhagens eretas e cinco rasteiros, com cinco repetições. As variáveis analisadas foram produtividades de vagens e de sementes. Em referência à análise estatística, realizaram-se análises de variância individual e conjunta e se adotou 0 teste de Tukey $(p<0,05)$ para comparação das médias dos tratamentos. A cultivar BR 1 foi utilizada como testemunha entre os genótipos eretos. A linhagem ereta LN-1B apresentou maior produção de vagens entre as demais do grupo, com $2.450 \mathrm{~kg} \mathrm{ha}^{-1}$, superando a média em $22 \%$ e a BR 1, em 11\%; a produtividade média em sementes situou-se em $1.665 \mathrm{~kg} \mathrm{ha}^{-1}$, correspondendo a $21 \%$ acima da média do grupo e $10 \%$ acima da BR 1. Entre as linhagens rasteiras as médias de produtividade em vagens e em sementes foram consideradas baixas nas condições estudadas destacando-se, contudo, LI-3, LI-5 e LI-1, que produziram em média entre $1.722 \mathrm{~kg} \mathrm{ha}^{-1}$ e $1.154 \mathrm{~kg} \mathrm{ha}^{-1}$, e superaram a média do grupo em 9.8 e 10\%, respectivamente.
\end{abstract}

Palavras-chave: Arachis hypogaea, tolerância a seca, melhoramento

\section{Yield of advanced lines of peanut under rainfed conditions in northeast Brazil}

\begin{abstract}
Eleven upright and runner peanut advanced lines were conducted conditions in four states located in the northeast region aiming to evaluate their pod and seed yield. Assays were carried out in the agricultural year 2005/2007 in the states of Pernambuco (Araripina and Parnamirim), Sergipe (Lagarto), Bahia (Cruz das Almas and Caetité) and Paraíba (Campina Grande and Itabaiana). A completely randomized block experimental design with five replications was used with 13 treatments (8 upright and 5 runner genotypes). The analyzed variables were pod and seed yields. Individual and joint variance analysis were carried out and Tukey $(p<0.05)$ test was used to compare treatments. The BR 1 cultivar was adopted as a control. The highest pod and seed yields were obtained by LN-1B, with 2,450 and 1,665 kg ha-1, respectively, overcoming the mean of upright lines in 22 and $21 \%$, and of the BR $1 \mathrm{cv}$. in 11 and $10 \%$. As to runner lines, the pod and seed yields were not satisfactory under the conditions, however, LI-3, LI-5 and LI-1 overcame the group, with 1,722 and 1,154 kg ha-1, corresponding to 9.8 and $10 \%$ over general mean, respectively.
\end{abstract}

Key words: Arachis hypogaea, drought tolerance, breeding

1 Embrapa Algodão, CP 174, CEP 58428-095, Campina Grande, PB. Fone: (83) 3182-4300. E-mail: caval@cnpa.embrapa.br 2 Embrapa Florestas, CP 319, CEP 83411-000, Colombo, PR. Fone: (41) 3675-5618. E-mail: gizelda@cnpf.embrapa.br

3 Empresa Baiana de D esenvolvimento Agropecuário, Rua Geraldo Suerdick s/n, CEP 44380-000, Cruz dasAlmas, BA. Fone: (75) 3621-1209. E-mail: cruzalmas@ebda.ba.gov.br 4 Instituto Agronômico de Pernambuco, Av. Gal. San Martin 1371, Bongi, CEP 50761-000, Recife, PE. Fone: (81) 3525-4392. E-mail: joao.coutinho@sdec.pe.gov.br 5 DEPA/UFRPE, Rua Dom Manoel de Medeiros s/n, Dois Irmãos, CEP 52171-900, Recife, PE. Fone: (81) 3320-6248. E-mail: pericles@depa.ufrpe.br 


\section{INTRODUÇÃO}

A região Nordeste é a segunda maior consumidora de amendoim e de seus derivados no Brasil. A produção obtida na faixa de $11.000 \mathrm{t}$ (IBGE, 2009), é insuficiente para atender à demanda regional, superior a $50.000 \mathrm{t}$, embora as condições edafoclimáticas das várias microrregiões sejam amplamente favoráveis ao desenvolvimento e estabelecimento da cultura.

A maioria das áreas de amendoim no Nordeste tem sido cultivada com cultivares eretas, em regime de sequeiro, cuja instabilidade das chuvas põe em risco, frequentemente, o desenvolvimento da lavoura, ocasionando a baixa produção (Nogueira \& Santos, 2000; Nogueira et al., 2006).

Os grãos das cultivares são indicados para o consumo in natura, que representa cerca de $50 \%$ do mercado de alimentos (Freitas et al., 2005). Contudo, com a atual expansão do mercado de castanhas e amêndoas tem surgido, recentemente, uma forte demanda por cultivares rasteiras, mais conhecidas como runners, por serem mais produtivas e indicadas para este segmento. As atuais cultivares em distribuição no Brasil não estão adaptadas para o semiárido nordestino, por serem tardias, com ciclo entre 120 e 140 dias, podendo chegar a até 150 dias (Santos et al., 2005), o que tem reduzido a competitividade do amendoim nordestino para segmento de confeitaria.

O programa de melhoramento de amendoim desenvolvido pela Embrapa Algodão tem, como principal objetivo, desenvolver cultivares produtivas, precoces e adaptadas ao manejo nas condições semiáridas do Nordeste brasileiro; para o alcance desses objetivos são testadas, anualmente, centenas de linhagens nacionais e internacionais em vários municípios nordestinos visando à identificação de genótipos promissores.

Das diversas populações anualmente geradas, $60 \%$ são destinados para atender ao mercado de consumo in natura, focalizando-se nas cultivares eretas e os $40 \%$ restantes para os mercados de confeitaria e oleoquímico, com maior enfoque para as cultivares rasteiras (Santos et al., 2005). A identificação de linhagens superiores que atendam a esses tipos de mercado e sejam adaptadas ao manejo em regime de sequeiro, é imprescindível para a alimentação do programa de melhoramento e posterior síntese de novas cultivares.

Apresenta-se, neste estudo, o desempenho produtivo de linhagens avançadas de amendoim, obtidas por meio de cruzamento entre cultivares nacionais e internacionais, cultivadas em condições de sequeiro em quatro estados do Nordeste brasileiro.

\section{MATERIAL E MÉTODOS}

As linhagens avaliadas neste estudo foram geradas na Embrapa Algodão, a partir de cruzamentos entre cultivares nacionais e estrangeiras, cedidas pelo Programa de Mejoramento Genetico y Manejo del Cultivo de Mani (Promani), da Argentina. Uma síntese da genealogia e dos descritores das linhagens se encontra na Tabela 1. Os progenitores Florunner (rasteiro) e Florida (erecto), Manfredi (424 e 407, ambos eretos) e 55437 (erecto), são de origem norte-americana, argentina e africana, respectivamente; os demais são nacionais de porte ereto, desenvolvidos pelo Instituto Agronômico de Campinas (IAC Poitara, IAC Tupã e IAC Oirã) e pertencentes à coleção de germoplasma da Embrapa Algodão (CNPA 51 e CNPA 01). Em comum, esses progenitores têm elevada produtividade e diferem no aspecto de adaptação e precocidade.

Os ensaios foram conduzidos em sete municípios de quatro Estados da região Nordeste: Araripina e Parnamirim (Sertão de Pernambuco), Cruz das Almas e Caetité (Recôncavo e Sertão baiano), Itabaiana e Campina Grande (Agreste e Brejo paraibano) e Lagarto (Tabuleiros Costeiros de Sergipe), de 2005 a 2007. Uma síntese das características do ambiente e período de plantio se encontra na Tabela 2.

O plantio foi realizado no início da estação chuvosa de cada município, em solos de textura variada (característica na Tabela 2), previamente corrigidos e adubados em função da necessidade da cultura. O plantio foi feito no espaçamento de $0,70 \times 0,20 \mathrm{~m}$, deixando-se duas plantas por cova. A parcela experimental foi constituída de três fileiras de $5 \mathrm{~m}$, utilizan-

Tabela 1. Genealogia e descritores das sementes das linhagens avaliadas neste estudo

\begin{tabular}{|c|c|c|c|c|c|c|c|}
\hline \multirow{2}{*}{ Genótipo } & \multirow{2}{*}{ Genealogia e origem dos progenitores } & \multirow{2}{*}{ Origem das linhagens } & \multirow{2}{*}{$\mathrm{HC}$} & \multicolumn{4}{|c|}{ Semente } \\
\hline & & & & Cor & Tamanho & Forma & No/vagem \\
\hline LI-1 & Florunner (EUA) x Manfredi 424 (Argentina) & Argentina & rasteiro & B & G & 1 & 2 \\
\hline $\mathrm{LI}-2$ & Manfredi 407 (Argentina) x Florunner & Argentina & rasteiro & $\mathrm{B}$ & G & 1 & 2 \\
\hline $\mathrm{LI}-3$ & Florunner x Manfredi 424 & Argentina & rasteiro & V & G & 1 & 2 \\
\hline $\mathrm{LI}-4$ & Manfredi 407 x Manfredi 424 & Argentina & rasteiro & V & G & 1 & 2 \\
\hline $\mathrm{LI}-5$ & Manfredi 407 x Manfredi 424 & Argentina & rasteiro & B & G & 1 & 2 \\
\hline LN-IV & IAC Poitara (São Paulo) x CNPA 92 AM (EUA) & Brasil & ereto & V & G & 1 & 3 \\
\hline $\mathrm{LN}-1 \mathrm{~B}$ & IAC Poitara x CNPA 92 AM (Florida) & Brasil & ereto & $\mathrm{B}$ & G & 1 & 3 \\
\hline LN-2 & 55437 (Senegal) x IAC Tupã (São Paulo) & Brasil & ereto & V & M & 2 & 3 \\
\hline LN-3 & 55437 x IAC Poitara & Brasil & ereto & V & M & 2 & 2 \\
\hline LN-4 & 55437 x CNPA 51 AM (São Paulo) & Brasil & ereto & B & M & 2 & 2 \\
\hline LN-5 & 55437 x CNPA 01 AM (Piauí) & Brasil & ereto & B & G & 2 & 2 \\
\hline LN-6 & 55437 AM x IAC Oirã (São Paulo) & Brasil & ereto & $\mathrm{B}$ & G & 1 & 2 \\
\hline BR-1 & Cultivar (Paraíba) & Brasil & ereto & V & $M$ & 2 & 4 \\
\hline
\end{tabular}

Legenda: LI - Linhagem Internacional; LN - Linhagem Nacional; HC - Hábito de Crescimento, Cor: B - Beje, V - Vermelha; Tamanho: G - Grande, M - Média; Forma: 1 - alongada, 2 - arredondada 
Tabela 2. Características do ambiente e período de plantio dos ensaios

\begin{tabular}{|c|c|c|c|c|c|c|c|c|}
\hline \multirow{2}{*}{ Local } & \multirow{2}{*}{$\begin{array}{c}\text { PPT } \\
(\mathrm{mm})\end{array}$} & \multirow{2}{*}{$\begin{array}{l}\text { UR } \\
(\%)\end{array}$} & \multirow{2}{*}{$\begin{array}{c}{ }^{\circ}{ }^{\circ} \mathrm{C} \\
\text { (média) }\end{array}$} & \multirow{2}{*}{$\begin{array}{c}\text { Altitude } \\
\text { (m) }\end{array}$} & \multicolumn{3}{|c|}{ Característica do Solo } & \multirow{2}{*}{ Plantio } \\
\hline & & & & & Tipo & Textura & $\mathrm{pH}$ & \\
\hline Parnamirim, PE & 478 & 80 & 29 & 397 & PVA & Argilosa & 6,5 & J aneiro \\
\hline Araripina, PE & 654 & 52 & 23 & 816 & LVA & Argilosa & 5,9 & J aneiro \\
\hline Lagarto, SE & 473 & 75 & 26 & 160 & REG & Arenosa & 5,3 & J unho \\
\hline Itabaiana, PB & 377 & 80 & 26 & 45 & REG & Franco-arenosa & 5,1 & Maio \\
\hline Campina Grande, PB & 348 & 83 & 23 & 548 & REG & Franco-arenosa & 6,4 & Abril \\
\hline Crus das Almas, BA & 501 & 86 & 23 & 220 & REG & Franco-arenosa & 5,5 & Maio \\
\hline Caetité, BA & 552 & 83 & 24 & 826 & REG & Franco-arenosa & 4,7 & Dez \\
\hline
\end{tabular}

PPT - Precipitação total registrada durante o ciclo; UR - umidade relativa do ar; T - temperatura

do-se a central como área útil $\left(3,5 \mathrm{~m}^{2}\right)$. Adotou-se o delineamento experimental em blocos ao acaso, com 13 tratamentos (12 linhagens mais a BR 1, como testemunha) e 5 repetições.

O manejo da cultura seguiu de acordo com o recomendado por Santos et al. (2006). As variáveis analisadas foram produtividades de vagens e de sementes. Realizaram-se, a análise estatística, as análises de variância individual e conjunta utilizando-se o programa SAS; para comparação das médias dos tratamentos utilizou-se o teste de Tukey $(\mathrm{P} 0,05)$.

\section{RESULTADOS E DISCUSSÃO}

As médias registradas para produtividade das vagens e das sementes nas linhagens eretas e rasteiras e o quadrado médio dos tratamentos, se encontram, respectivamente, nas Tabelas 3 e 4. Observou-se diferença estatística significativa entre os tra- tamentos, em todos os locais estudados, para ambas as variáveis; a exceção foi observada nas médias das plantas rasteiras conduzidas em Itabaiana, PB, para rendimento em vagens e na média conjunta, para rendimento em sementes.

A média conjunta das linhagens eretas foi superior à das rasteiras, tanto para produtividade em vagens quanto de sementes destacando-se, entre todas as linhagens avaliadas, a ereta LN-1B, com $2.450 \mathrm{~kg} \mathrm{ha}^{-1}$ em vagens e $1.665 \mathrm{~kg} \mathrm{ha}^{-1}$ em sementes, superando a média das linhagens do mesmo grupo em 22 e $21 \%$ (Tabelas 3 e 4). Considerando-se a média da BR 1, a produtividade de vagens e sementes da LN-1B foi superior em à da BR 1, em 11 e 10\%, valores esses significativos quando se considera que a BR 1 é a cultivar de maior aceitação para o semiárido, em virtude da alta produtividade e adaptação (Santos et al., 1999; Coutinho et al., 2002; Gomes et al., 2007), e reflete o elevado valor genético desta linhagem, herdada pela contribuição da paulista ereta

Tabela 3. Análise individual e conjunta para produtividade de vagens das linhagens de amendoim, em quatro Estados do Nordeste

\begin{tabular}{|c|c|c|c|c|c|c|c|c|}
\hline \multirow{3}{*}{ Genótipo } & \multicolumn{8}{|c|}{ Rendimento em vagem (kg ha-1) } \\
\hline & \multicolumn{2}{|c|}{$\mathrm{PE}$} & \multirow{2}{*}{$\begin{array}{c}\text { SE } \\
\text { Lagarto }\end{array}$} & \multicolumn{2}{|c|}{ PB } & \multicolumn{2}{|c|}{ BA } & \multirow{2}{*}{$\begin{array}{c}\text { Média } \\
\text { Conjunta }\end{array}$} \\
\hline & Parnamirim & Araripina & & Itabaiana & C.Grande & C.Almas & Caetité & \\
\hline \multicolumn{9}{|l|}{ Ereto } \\
\hline LN-1B & 3520,0 a & $4360,0 \mathrm{a}$ & $1766,0 \mathrm{~b}$ & $1059,0 \mathrm{C}$ & $1709,0 a b$ & $2494,0 \mathrm{a}$ & $2246,0 \mathrm{a}$ & 2450,0 a \\
\hline $\mathrm{LN}-2$ & $2887,0 a b$ & 2309,0 ef & $1488,0 \mathrm{C}$ & $1313,0 \mathrm{a}$ & $1846,0 \mathrm{a}$ & $1326,0 \mathrm{C}$ & $2099,0 a b$ & $1895,0 \mathrm{bc}$ \\
\hline BR-1 & $2840,0 \mathrm{ab}$ & 3584,0 bc & $1981,0 \mathrm{a}$ & $1453,0 \mathrm{a}$ & 1594,0 bc & $2308,0 \mathrm{a}$ & $1636,0 \mathrm{~b}$ & 2199,0 a \\
\hline LN-4 & $2635,0 a b$ & $4024,0 a b$ & $1647,0 \mathrm{bc}$ & $1160,0 \mathrm{bc}$ & $1050,0 \mathrm{~d}$ & $2336,0 \mathrm{a}$ & $2185,0 a b$ & $2148,0 a b$ \\
\hline LN-5 & $2120,0 \mathrm{~b}$ & $2991,0 \mathrm{~cd}$ & $1377,0 \mathrm{~d}$ & $1044,0 \mathrm{C}$ & $1405,0 \mathrm{C}$ & $2250,0 \mathrm{a}$ & $2039,0 a b$ & $1889,0 \mathrm{C}$ \\
\hline LN-3 & $2111,0 \mathrm{~b}$ & $1647,0 \mathrm{f}$ & 1231,0 de & $1041,0 \mathrm{c}$ & $1696,0 \mathrm{ab}$ & $1804,0 \mathrm{~b}$ & $1711,0 a b$ & $1606,0 \mathrm{~d}$ \\
\hline LN-1V & $2108,0 \mathrm{~b}$ & $3751,0 a b$ & $915,0 \mathrm{f}$ & $1078,0 \mathrm{bc}$ & $1696,0 \mathrm{ab}$ & $2220,0 \mathrm{a}$ & $1636,0 \mathrm{~b}$ & $1915,0 \mathrm{~b}$ \\
\hline LN-6 & $2100,0 \mathrm{~b}$ & 2901,0 de & $1203,0 \mathrm{e}$ & $1012,0 \mathrm{C}$ & $1169,0 \mathrm{~d}$ & $2399,0 \mathrm{a}$ & $2058,0 a b$ & $1834,0 \mathrm{C}$ \\
\hline QMTE & 1130532* & $154725^{* *}$ & 586988* & $124271^{* *}$ & $404606 * *$ & 1029209* & 313635* & \\
\hline CV (\%) & 11,28 & 8,24 & 9,36 & 10,12 & 13,44 & 12,26 & 9,67 & \\
\hline Média & 2685 & 3196 & 1451 & 1145 & 1521 & 2111 & 1951 & 2008 \\
\hline DMS & 1003 & 678 & 200 & 243 & 223 & 327 & 605 & 468 \\
\hline \multicolumn{9}{|l|}{ Rasteiro } \\
\hline LI-3 & $3209,0 \mathrm{a}$ & $3120,0 \mathrm{a}$ & $1006,0 \mathrm{~b}$ & 922,0 a & $1485,0 \mathrm{~b}$ & $1536,0 \mathrm{bc}$ & $1227,0 \mathrm{~b}$ & 1786,0 a \\
\hline LI-5 & $2673,0 \mathrm{a}$ & $2656,0 a b$ & $920,0 \mathrm{~b}$ & 716,0 a & $1034,0 \mathrm{C}$ & $1750,0 a b$ & 1916,0 a & $1666,0 \mathrm{ab}$ \\
\hline LI-2 & $2456,0 \mathrm{a}$ & $1738,0 \mathrm{c}$ & $1301,0 \mathrm{a}$ & $757,0 \mathrm{a}$ & $1075,0 \mathrm{C}$ & $1420,0 \mathrm{C}$ & $1201,0 \mathrm{~b}$ & $1421,0 \mathrm{~b}$ \\
\hline LI-1 & $2446,0 \mathrm{a}$ & $2359,0 \mathrm{~b}$ & $865,0 \mathrm{~b}$ & $825,0 \mathrm{a}$ & $1948,0 \mathrm{a}$ & $1918,0 \mathrm{a}$ & $1642,0 a b$ & $1715,0 \mathrm{a}$ \\
\hline LI-4 & $1479,0 \mathrm{~b}$ & $2169,0 \mathrm{bc}$ & $829,0 \mathrm{~b}$ & $730,0 \mathrm{a}$ & $1150,0 \mathrm{C}$ & $1302,0 \mathrm{C}$ & $1146,0 \mathrm{~b}$ & $1258,0 \mathrm{c}$ \\
\hline QMTR & 1568079* & $1345734 * *$ & 179364* & $36133^{\text {ns }}$ & 737388** & 309786* & $568750 * *$ & \\
\hline CV (\%) & 15,83 & 18,42 & 19,68 & 18,76 & 15,74 & 12,63 & 10,37 & \\
\hline Média & 2453 & 2408 & $984 \mathrm{~b}$ & $790 \mathrm{~b}$ & 1338 & 1585 & 1426 & 1569 \\
\hline DMS & 957 & 604 & 178 & 216 & 199 & 312 & 539 & 429 \\
\hline
\end{tabular}

Médias seguidas da mesma letra não diferem estatisticamente entre si pelo teste de Tukey, a $5 \%$ de probabilidade; QMTE - quadrado médio dos tratamentos eretos; QMTR, quadrado médio dos Tratamentos rasteiros, ${ }^{*}$ - significativo $(\mathrm{P} 0,05),{ }^{* *}-$ significativo $(\mathrm{P} 0,01)$, ns - não significativo pelo teste $\mathrm{F}$ 
IAC Poitara, de grãos grandes e longos e a norte-americana rasteira, Florida, de grãos grandes e tolerantes às doenças de folhagens (Tabela 1).

A linhagem LN-4, oriunda do cruzamento entre a africana e resistente à seca 55 437, e a paulista de grãos grandes, CNPA $51 \mathrm{AM}$, ambas de porte ereto, também foram produtivas e situaram no mesmo grupo estatístico da BR 1 (Tabela 3). As produtividades em vagens e em sementes desta linhagem se situaram em $2.148 \mathrm{~kg} \mathrm{ha}^{-1}$ e $1.468 \mathrm{~kg} \mathrm{ha}^{-1}$, superando a média do grupo em 7 e $6 \%$, respectivamente.

Entre os locais estudados, contudo, os melhores resultados das linhagens eretas foram conseguidos no sertão pernambucano, em Parnamirim e Araripina (Tabelas 3 e 4), beneficiados pelas condições climáticas e pelas características dos solos, PVA e LVA argilosos, respectivamente (Tabela 2). As linhagens nacionais LN-1B e LN 4 mostraram melhor desempenho para a produção de vagens e sementes com rendimentos médios de $3.635 \mathrm{~kg} \mathrm{ha}^{-1}$ e $2.544 \mathrm{~kg} \mathrm{ha}^{-1}$, respectivamente, superiores aos das médias do grupo, em 24 e $23 \%$. Considerando-se, contudo, a média da BR 1 no Estado, a média das linhagens superou o da cultivar em apenas $13 \%$, tanto para produção de vagens quanto de sementes.

Em Sergipe nenhuma linhagem ereta superou a BR 1 para produção de vagem ou de sementes e, na Paraíba, apenas a linhagem $\mathrm{LN}-2$ produziu $18 \%$ acima da média obtida no Estado e 4\%, acima da BR 1, na produção de vagens.

Na Bahia, as linhagens LN-1B, LN-4, LN-5 e LN-6 se mantiveram no mesmo grupo estatístico nos dois locais, produzindo cerca de $2.250 \mathrm{~kg} \mathrm{ha}^{-1}$ em vagens, superando a mé- dia do grupo e da BR 1, em 11 e 14\%, respectivamente. Diferentemente à produção em sementes, no entanto, a LN-5 não se sobressaiu nos dois locais; a média das linhagens LN-1B, LN-4 e LN-6 se situou em $1.544 \mathrm{~kg} \mathrm{ha}^{-1}$ superior, portanto, à média e à BR 1, em 28 e $15 \%$, respectivamente.

Com relação às linhagens rasteiras se destacaram, no aspecto conjunto, LI-3, LI-5 e LI-1, com rendimento médio de $1.722 \mathrm{~kg} \mathrm{ha}^{-1}$ para produtividade de vagens, superando a média entre as demais do grupo, em 10\% (Tabela 3). A contribuição da carga genética da cultivar Argentina Manfredi 424 deve ter influenciado este rendimento, uma vez que as cultivares Florunner e Manfredi 407 são mais responsivas em ambientes favoráveis (Godoy et al., 1999, 2003). Para produção de sementes não houve diferença estatística entre as linhagens, que apresentaram média de $1.046 \mathrm{~kg} \mathrm{ha}^{-1}$ (Tabela 4).

No Estado de Pernambuco as linhagens mais indicadas foram LI-3 e LI-5, com média de 3.165 e $2.665 \mathrm{~kg} \mathrm{ha}^{-1}$, respectivamente; para o perímetro irrigado do Vale do São Francisco, onde a demanda por amendoim rasteiro tem crescido anualmente, esses materiais se apresentam como boa alternativa considerando-se as condições de luminosidade e disponibilidade hídrica, características da região.

Em Sergipe e na Paraíba, apesar do rendimento baixo, as linhagens LI-2 e LI-1 apresentaram maior produtividade em vagens considerando-se a média do grupo, de $1.301 \mathrm{e}$ $1.387 \mathrm{~kg} \mathrm{ha}^{-1}$, respectivamente, enquanto na Bahia se destacaram LI-5 e LI-1, com médias de 1.833 e $1.780 \mathrm{~kg} \mathrm{ha}^{-1}$.

Os resultados obtidos com as linhagens rasteiras nesses ensaios indicam que, de forma geral, a condição de manejo a

Tabela 4. Analise individual e conjunta para produtividade média de sementes ( $\mathrm{kg} \mathrm{ha-1)}$ ) das linhagens de amendoim, em quatro Estados do Nordeste

\begin{tabular}{|c|c|c|c|c|c|c|c|c|}
\hline \multirow{3}{*}{ Genótipo } & \multicolumn{8}{|c|}{ Rendimento em sementes $\left(\mathrm{kg} \mathrm{ha}^{-1}\right)$} \\
\hline & \multicolumn{2}{|c|}{$\mathrm{PE}$} & \multirow{2}{*}{$\begin{array}{c}\text { SE } \\
\text { Lagarto }\end{array}$} & \multicolumn{2}{|c|}{ PB } & \multicolumn{2}{|c|}{ BA } & \multirow{2}{*}{$\begin{array}{c}\text { Média } \\
\text { Conjunta }\end{array}$} \\
\hline & Parnamirim & Araripina & & Itabaiana & C.Grande & C.Almas & Caetité & \\
\hline \multicolumn{9}{|l|}{ Ereto } \\
\hline LN-1B & 2464,0 a & 3052,0 a & $1165,0 \mathrm{~b}$ & $741,0 \mathrm{C}$ & $1024,0 \mathrm{~b}$ & $1754,0 \mathrm{a}$ & $1453,0 a b$ & $1665,0 \mathrm{a}$ \\
\hline $\mathrm{LN}-2$ & $2021,0 \mathrm{ab}$ & $1616,0 \mathrm{c}$ & $1018,0 \mathrm{bc}$ & $919,0 \mathrm{ab}$ & $1292,0 \mathrm{a}$ & $996,0 \mathrm{~d}$ & $1469,0 a b$ & $1333,0 \mathrm{~b}$ \\
\hline BR-1 & $1988,0 \mathrm{ab}$ & $2509,0 \mathrm{bcd}$ & $1335,0 \mathrm{a}$ & $1017,0 \mathrm{a}$ & $1005,0 \mathrm{~b}$ & $1646,0 \mathrm{a}$ & $1083,0 \mathrm{bc}$ & $1512,0 \mathrm{a}$ \\
\hline LN-4 & $1844,0 a b$ & $2817,0 a b$ & $1065,0 \mathrm{bc}$ & 814,0 a & $615,0 d$ & $1584,0 a b$ & $1540,0 \mathrm{a}$ & $1468,0 a b$ \\
\hline LN-5 & $1484,0 \mathrm{~b}$ & $2206,0 \mathrm{~cd}$ & $930,0 \mathrm{~cd}$ & $730,0 \mathrm{bc}$ & $900,0 \mathrm{bc}$ & $1323,0 \mathrm{C}$ & $1413,0 a b$ & $1095,0 \mathrm{bc}$ \\
\hline LN-3 & $1478,0 \mathrm{~b}$ & $1153,0 \mathrm{f}$ & $830,0 \mathrm{~d}$ & $729,0 \mathrm{C}$ & $1034,0 \mathrm{~b}$ & $1265,0 \mathrm{~b}$ & $1149,0 \mathrm{abc}$ & $1091,0 \mathrm{bc}$ \\
\hline LN-1V & $1368,0 \mathrm{c}$ & $2626,0 \mathrm{abc}$ & $608,0 \mathrm{e}$ & $730,0 \mathrm{c}$ & $1018,0 \mathrm{~b}$ & $1326,0 \mathrm{C}$ & $846,0 \mathrm{C}$ & $833,0 d$ \\
\hline LN-6 & $1321,0 \mathrm{C}$ & $2031,0 \mathrm{~cd}$ & $838,0 \mathrm{~d}$ & $709,0 \mathrm{c}$ & $749,0 \mathrm{~cd}$ & 1686,0 a & $1446,0 a b$ & $1065,0 \mathrm{bc}$ \\
\hline QMTE & $554040 *$ & 2020615* & $62155^{* *}$ & $208587^{* *}$ & $299483 * *$ & $436431 *$ & $251726 *$ & $554040 *$ \\
\hline CV (\%) & 10,12 & 7,26 & 15,54 & 13,26 & 14,74 & 12,62 & 10,76 & \\
\hline Média & 1880 & 2251 & 800 & 955 & 1300 & 1488 & 973 & 1378 \\
\hline DMS & 702 & 494 & 165 & 239 & 417 & 278 & 167 & \\
\hline \multicolumn{9}{|l|}{ Rasteiro } \\
\hline LI-3 & $2247,0 \mathrm{a}$ & $2184,0 \mathrm{a}$ & $604,0 \mathrm{~b}$ & 646,0 a & $881,0 \mathrm{~b}$ & $928,0 a b c$ & $859,0 b c$ & 1193,0 a \\
\hline LI-5 & $1871,0 \mathrm{a}$ & $1859,0 a b$ & $558,0 \mathrm{~b}$ & $501,0 \mathrm{a}$ & $629,0 \mathrm{C}$ & $1025,0 a b$ & $1273,0 \mathrm{a}$ & $1102,0 \mathrm{a}$ \\
\hline LI-2 & $1719,0 \mathrm{a}$ & $1217,0 \mathrm{C}$ & $811,0 \mathrm{a}$ & $430,0 \mathrm{a}$ & $629,0 \mathrm{C}$ & 828,0 bc & $803,0 b c$ & 919,0 a \\
\hline LI-1 & $1712,0 \mathrm{a}$ & $1651,0 \mathrm{bc}$ & $520,0 \mathrm{~b}$ & $577,0 \mathrm{a}$ & $1392,0 \mathrm{a}$ & $1147,0 a b$ & $1173,0 a b$ & $1167,0 \mathrm{a}$ \\
\hline LI-4 & $1035,0 \mathrm{~b}$ & $1518,0 \mathrm{bc}$ & $485,0 \mathrm{~b}$ & 511,0 a & $756,0 \mathrm{bc}$ & $754,0 \mathrm{C}$ & $768,0 \mathrm{C}$ & $832,0 \mathrm{a}$ \\
\hline QMTR & 769164* & 659619* & $17776 *$ & $501491^{\mathrm{ns}}$ & 267643* & $121626 *$ & $82244 * *$ & \\
\hline CV (\%) & 12,88 & 10,40 & 17,36 & 10,87 & 13,32 & 18,22 & 14,77 & \\
\hline Média & 1717 & 1686 & 596 & 553 & 857 & 936 & 975 & 1046 \\
\hline DMS & 670 & 440 & 148 & 147 & 213 & 265 & 371 & \\
\hline
\end{tabular}

Médias seguidas da mesma letra não diferem estatisticamente entre si pelo teste de Tukey, a $5 \%$ de probabilidade; QMTE - quadrado médio dos tratamentos eretos; QMTR, quadrado médio dos Tratamentos rasteiros, ${ }^{*}$ - significativo $(\mathrm{P} 0,05), * *$ - significativo $(\mathrm{P} 0,01),{ }^{\text {ns }}$ - não significativo pelo teste $\mathrm{F}$ 
elas imposta não foi a mais adequada e necessita de otimização, em especial quanto à fertilidade e disponibilidade hídrica, para que se permita a melhor expressão produtiva das linhagens (Godoy et al., 2003, Pereira et al., 2008). Na região Sudeste do País, onde a disponibilidade de água e as características do solo favorecem o desenvolvimento das cultivares de ciclo longo, a produtividade dos materiais rasteiros é geralmente elevada, situando-se entre 3.700 e $5.100 \mathrm{~kg} \mathrm{ha}^{-1}$, com rendimento em sementes na faixa de 70\% (Godoy et al., 2003; Oliveira et al., 2006). Por outro lado, genótipos rasteiros possuem ciclo mais longo, em torno de 120-150 dias, e não toleram veranicos na estação de floração, fato comum de ocorrer em várias microrregiões do Nordeste.

Távora et al. (2002) testaram oito genótipos de amendoim de porte ereto e rasteiro no Ceará e concluíram que os rasteiros são mais instáveis e menos previsíveis enquanto os eretos atingem maior grau de adaptação sob condições limitadas e de irregularidade de disponibilidade hídrica. Portanto, para recomendação de linhagens rasteiras nesta região fazse necessária a condução de outros ensaios relacionados à otimização no manejo, focalizando-se na fertilidade do solo, densidade de plantio e disponibilidade hídrica, de modo a permitir que as linhagens expressem melhor sua capacidade de produção.

\section{CONCLUSÕES}

1. As linhagens nacionais $\mathrm{LN}-1 \mathrm{~B}$ é a mais indicada para o cultivo de sequeiro nas condições de manejo, nos ambientes estudados.

2. As linhagens rasteiras demonstraram baixa adaptação ao cultivo de sequeiro, carecendo de ensaios adicionais, de modo a otimizar o manejo e elevar a capacidade produtiva.

\section{LITERATURA CITADA}

Coutinho, J. L. B.; Tavares, J. A.; Santos, V. F.; Santos, R. C.; Carvalho Neto, A. Estabilidade e adaptabiidade de genótipos de amendoim (Arachis hypogaea L.) na chapada do Araripe, em Pernambuco. Pesquisa Agropecuária Pernambucana, v.13, p.17-24, 2002.

Freitas, S. M.; Martins, S. S.; Nomi, A. K; Campos, A. F. Evolução do mercado brasileiro de amendoim. In: Santos, R. C. (ed.). O Agronegócio do amendoim no Brasil. Campina Grande: Embrapa Algodão; Brasília: Embrapa Informação Tecnológica, 2005. p.15-44.
Godoy, I. J.; Moraes, S. A.; Kasai, F. S.; Martins, H. L. M.; Pereira, J. C. V. N. A.; Moraes, A. R. A.; Teixeira, J. P. F. Cultivares IAC de amendoim. O Agronômico, v.55, n.1, p.26-29, 2003.

Godoy, I. J.; Moraes, S. A.; Siqueira, A. L. M.; Pereira, J. C. V. N. A.; Martins, P. H. L. M.; Paulo, E. M. Produtividade, estabilidade e adaptabilidade de cultivares de amendoim em três níveis de controle de doenças foliares. Pesquisa Agropecuária Brasileira, v.34, n.7, p.1183-1191, 1999.

Gomes, L. R.; Santos, R. C.; Anunciação Filho, C. J.; Melo Filho, P. A. Adaptabilidade e estabilidade fenotípica de genótipos de amendoim de porte ereto. Pesquisa Agropecuária Brasileira, v.42, n.7, p.985-989, 2007.

IBGE - Instituto Brasileiro de Geografia e Estatística. Produção Agrícola. http://www.ibge.gv.br/home/estatistica. 23 Dez. 2009.

Nogueira, R. J. M. C.; Melo Filho, P. A.; Carvalho, R.; Santos, R. C. Comportamento estomático e potencial da água da folha em amendoim cv. BRS 151-L7 submetido a estresse hídrico. Revista de Oleaginosas e Fibrosas, v.10, p.985-991, 2006.

Nogueira, R. J. M. C.; Santos, R. C. Alterações fisiológicas no amendoim submetido ao estresse hídrico. Revista Brasileira de Engenharia Agrícola Ambiental, v.4, n.1, p.41-45, 2000.

Oliveira, E. J.; Godoy, I. J.; Moraes, A. R. A.; Martins, A. L. M.; Pereira, J. C. V. N. A.; Bortoletto, N.; Kasai, F. S. Adaptabilidade e estabilidade de genótipos de amendoim de porte rasteiro. Pesquisa Agropecuária Brasileira, v.41, n.8, p.1253-1260, 2006.

Pereira, J. W. L., Melo Filho, P. A.; Silva, F. A. C., Santos, R. C. Variabilidade genética de acessos de amendoim do tipo runner com base em marcadores RAPD. Revista Brasileira de Oleaginosas e Fibrosas, v.12, n.1, p.35-40, 2008.

Santos, R. C.; Farias, F. J. C.; Rêgo, G. M.; Silva, A. P. G.; Ferreira Filho, J. R.; Vansconcelos, O. L. Estabilidade fenotípica de cultivares de amendoim avaliadas na região Nordeste do Brasil. Ciência \& Agrotecnologia, v.23, p.808-812, 1999.

Santos, R. C.; Godoy, I. J.; Favero, A. P. Melhoramento do amendoim. In: Santos, R. C. (ed.) O agronegócio do amendoim no Brasil. Campina Grande: Embrapa Algodão; Brasilia: Embrapa Informações Tecnológicas, 2005. p.23-190.

Santos, R. C.; Rego, G. M.; Santos, C. A.; Melo Filho, P. A.; Silva, A. P. G.; Gondim, T. M. S; Suassuna, T. F. Recomendações técnicas para o cultivo do amendoim. Campina Grande: EMBRAPA, 2006. 6p. Circular Técnica, n.102

Távora, F. J. A. F.; Silva, F. P.; Melo, F. I. O.; Pitombeira, J. B.; Costa Neto, F. V.; Vieira. F. Yield adaptability and stability of peanut genotypes estimated under different environments. Ciência Agronômica, v.33, p.10-14, 2002. 